\section{Rotation of Bodies with Dielectric Surfaces in Electrostatic Fields.}

REFERRING to Dr. S. W. Richardson's letter in NATURE of Feb. 12 on the rotation of dielectric bodies in electrostatic fields shown by Mr. L. G. Vedy and Mr. G. Gowlland, at the recent exhibition of the Physical Society, it may be of interest to point out that at the Exhibition in $1896 \mathrm{I}$ showed two experiments which seem to bear on the subject. The first was with a highly dried dielectric liquid. If a spherical electrode is fixed some $5 \mathrm{~mm}$. or $6 \mathrm{~mm}$ above the surface and charged from one pole of a Wimshurst, the other pole being earthed, the liquid quietly rises up in a conical form, attaches itself to the electrode, and remains suspended while the field is maintained.

If this same liquid is then exposed to the atmosphere or otherwise absorbs a small percentage of moisture, on repeating the experiment-while there is still a tendency to lift the liquid--there is an even stronger one to depress it in places resulting in great turbulence, hollows, and sometimes whirling motions, all of which vanish if the liquid is again dried.

In a letter in NATuRe for April 5, 1924, I showed that the properties called 'absorption' in dielectrics at ordinary frequencies are also due to small percentages of moisture and can be greatly altered by varying this. It would, therefore, appear that with dielectric liquids these effects are also due to 'ab. sorption'.

If a short bar of a good dielectric, but showing some absorption, is placed between two electrodes, but with air-gaps, and a fairly strong field is maintained in a dried atmosphere for some time and it is then, with insulating tongs, brought near an electroscope, the two ends show opposite charges, and these take time to die out. This action is separate from the opposite induced polarity of the mass of the molecules of the dielectric, which is instantaneous, and disappears instantaneously when the field is removed. This latter action appears to take no part in the rotations which are due to interaction between the field and moisture in the dielectric, or to its ' absorption'.

In the letter referred to I mentioned that to produce the absorptive effects it was not enough to assume the dielectric constant of water as 80 , but that the only conclusion was that when small percentages of moisture are occluded in dielectrics, much, at any rate, must exist in an ionised form, when the reaction of the separated ions with an outside field would be much more powerful.

From observation of the experiments I gathered that rotation only occurs when brush discharges of sufficient magnitude pass between the electrodes and the rotating body.

If a sheet of a good dielectric is held near such a charged electrode, and is then bronght to an electroscope, the surface of the dielectric shows a charge, but it is of the same polarity as the electrode producing it, instead of having the opposite induced polarity which it would have had if the dielectric had been held at a greater distance.

Now when a dielectric having in it ionised moisture is placed in an electrical field, the negative ions are strongly attracted towards the positive electrode, and vice versa, and these ions tend to be drawn through the dielectric and to accumulate under the opposite surfaces. Primarily this would produce opposite induced polarity; but if the field is sufficiently strong there is evidence that some of the opposite ions are actually pulled out of the dielectric and drawn to the electrode. This leaves the dielectric opposite the electrode with a free charge, but of the same sign as the electrode, causing strong repulsion.
To start the rotations there must be initial movement of the dielectric or some want of symmetry. The rotation takes place either clockwise, or counterclockwise, depending on the direction of the initial impulse.

Thus if a block of dielectric is placed in a strong field the electrodes extract opposite ions from the two opposed surfaces and leave each with a strong charge of the same sign as the opposite electrode. When the dielectric is given a slight movement one way or the other, these charges are repelled by the electrodes and both unite in turning the dielectric in the same direction. Now these charges in the dielectric, if the dielectric is what is called 'good,' or has a sufficiently high resistance, take some time to die away. They are continually renewed as fresh surfaces of the dielectric pass the electrodes, and, on the other hand, as the rotation carries each charge round towards the next electrode the charge will be of opposite sign and therefore will be attracted until it gets under the electrode. As it comes under the second electrode the attraction will draw the ions of opposite polarity out of the dielectric and again leave it with a charge of the same sign, again producing repulsion, all the actions thus uniting to produce motion in the same direction.

Surface moisture will also give rise to the same actions if the film is sufficiently thin. If, however, the film forms a layer of low enough resistance, surface conduction will neutralise the opposite charges on the dielectric and thus reduce or stop the rotation. This was observable in some of the experiments. For example, a metal cylinder wrapped with paper would not rotate until the paper was dried, and the same inability to rotate occurred with a glass beaker before it was surface dried.

Speaking generally, this seems to be the explanation of these interesting demonstrations.

I have been investigating actions of this character during the last two years under conditions calculated to show their causes more clearly; the results, it is hoped, will be published shortly.

35 Holland Villas Road,

G. L. Addenbrooke.

Kensington, W., Feb. 21.

\section{The Effect of Intense Light on the Energy Levels of Atoms.}

IN 1923, Bohr (Z.f. Phys., I 3, p. 117; 1923) directed attention to the fact that the usual formulæ of the older quantum theory might not be strictly applicable to atoms which are subjected to intense radiation fields of high frequency, such as those of ordinary light. Schrödinger (see particularly equation $\mathbf{1 6}$, Ann.d. Phys., 8r, p. $109 ; 1926$ ) has formulated the differential equation for the $\psi$-function of an atom exposed to a harmonic electric force, and from this the allowed values of the quantity $E$ appearing in the equation may be determined. In such a case, however, the physical interpretation of $E$ is doubtful, though it seems highly probable that it represents the (average) energy of the disturbed atom. Thus we cannot be certain that the equation $E_{1} E_{2}=h \nu$ is correct for calculating the frequencies emitted by such an atom. Whatever be the final formulation of the theory, it seems reasonable to suppose that the energy levels of atoms in sources at very high temperatures may be modified by the electric and magnetic fields of the radiation from the source. itself.

Mr. W. Kuhn (Z.f. Phys., 38, p. 440; 1926) devised an interesting experiment for detecting changes in the energy levels of sodium atoms illuminated with 